\title{
Which factors prognosticate rotational instability following lumbar laminectomy?
}

\author{
Arno Bisschop • Idsart Kingma • Ronald L. A. W. Bleys • \\ Albert J. van der Veen • Cornelis P. L. Paul • \\ Jaap H. van Dieën · Barend J. van Royen
}

Received: 6 March 2013/Revised: 17 July 2013/Accepted: 6 September 2013/Published online: 17 September 2013

(C) Springer-Verlag Berlin Heidelberg 2013

\begin{abstract}
Purpose Reduced strength and stiffness of lumbar spinal motion segments following laminectomy may lead to instability. Factors that predict shear biomechanical properties of the lumbar spine were previously published. The purpose of the present study was to predict spinal torsion biomechanical properties with and without laminectomy from a total of 21 imaging parameters.

Method Radiographs and MRI of ten human cadaveric lumbar spines (mean age 75.5, range 59-88 years) were obtained to quantify geometry and degeneration of the motion segments. Additionally, dual X-ray absorptiometry (DXA) scans were performed to measure bone mineral content and density. Facet-sparing lumbar laminectomy was performed either on L2 or L4. Spinal motion segments were dissected (L2-L3 and L4-L5) and tested in torsion, under $1,600 \mathrm{~N}$ axial compression. Torsion moment to
\end{abstract}

\footnotetext{
A. Bisschop · C. P. L. Paul · B. J. van Royen $(\square)$

Department of Orthopedic Surgery, Research Institute MOVE, VU University Medical Center, De Boelelaan 1117, P.O. Box 7057, 1081 HV Amsterdam, The Netherlands e-mail: bj.vanroyen@vumc.nl

I. Kingma $\cdot$ J. H. van Dieën

Research Institute MOVE, Faculty of Human Movement Sciences, VU University Amsterdam, Van der Boechorststraat 7, 1081 BT Amsterdam, The Netherlands
}

R. L. A. W. Bleys

Division of Surgical Specialties, Department of Anatomy, University Medical Center Utrecht, P.O. Box 85060,

3508 AB Utrecht, The Netherlands

A. J. van der Veen

Department of Physics and Medical Technology, VU University

Medical Center, De Boelelaan 1117, P.O. Box 7057,

1081 HV Amsterdam, The Netherlands failure (TMF), early torsion stiffness (ETS, at 20-40\% TMF) and late torsion stiffness (LTS, at 60-80\% TMF) were determined and bivariate correlations with all parameters were established. For dichotomized parameters, independent-sample $t$ tests were used.

Results Univariate analyses showed that a range of geometric characteristics and disc and bone quality parameters were associated with torsion biomechanical properties of lumbar segments. Multivariate models showed that ETS, LTS and TMF could be predicted for segments without laminectomy ( $r^{2}$ values $0.693,0.610$ and 0.452 , respectively) and with laminectomy ( $r^{2}$ values $0.952,0.871$ and 0.932 , respectively), with DXA-derived measures of bone quality and quantity as the main predictors.

Conclusions Vertebral bone content and geometry, i.e. intervertebral disc width, frontal area and facet joint tropism, were found to be strong predictors of ETS, LTS and TMF following laminectomy, suggesting that these variables could predict the possible development of postoperative rotational instability following lumbar laminectomy. Proposed diagnostic parameters might aid surgical decision-making when deciding upon the use of instrumentation techniques.

Keywords Torsion biomechanics - Decompression . Laminectomy $\cdot$ Spinal stenosis and diagnostics

\section{Introduction}

Prediction of residual strength and stiffness of a spinal segment after laminectomy is useful for a surgeon to decide whether or not to use instrumented fusion techniques. Shear biomechanical properties after laminectomy could be predicted from imaging data, which may support such 
decision-making [4]. However, in daily practice the spine is also loaded in torsion [3]. Torsion loads may cause and progress disc degeneration and may even cause failure of a segment $[1,7,8,10]$. Previously, it has been shown in an in vitro experiment that laminectomy results in a substantial decrease of torsion stiffness and torsion moment to failure (TMF) of lumbar spinal segments [3]. For shear loads, the biomechanical behaviour of a spinal motion segment following laminectomy has been shown to depend on disc degeneration, facet joint degeneration, Modic changes, Schmorl's nodes, intervertebral disc and pedicle geometry, and facet joint angles [4]. This may also hold for torsion strength and stiffness following laminectomy. If true, such variables, which can be assessed based on imaging prior to surgery, may aid surgical decision-making on the need for instrumented stabilization of the spine during surgery.

Therefore, the aim of this study is to establish, as was done previously for shear biomechanics, the correlation between a broad range of spinal properties assessed by 21 imaging parameters and torsion biomechanical properties with and without laminectomy. To this end, we studied in vitro torsion stiffness and TMF of lumbar spinal segments either untreated or following facet-sparing laminectomy. Ten segments remained untreated (five times L2L3 and five times L4-L5) and ten segments were treated with laminectomy (five times L2-L3 and five times L4L5). We hypothesized that multiple independent variables, together, determine torsion biomechanics of a lumbar spinal segment either intact or treated with laminectomy.

\section{Methods}

\section{Specimens}

Ten lumbar spines (L1-L5) were harvested from freshly frozen $\left(-20{ }^{\circ} \mathrm{C}\right.$ ) human cadavers (mean age 75.5 , range 59-88 years). The bodies were donated to the Department of Anatomy of the UMC Utrecht by last will in accordance with the Dutch legislation and were destined for medical education and research. Body handling was done according to the guidelines of the Department of Anatomy. None of the deceased subjects had any history of spinal injury, spinal surgery or spinal metastatic disease. The spines were thawed before imaging and biomechanical testing. Before imaging, also excessive soft tissues were carefully removed, keeping the anterior and posterior longitudinal ligaments as well as the facet joints intact.

\section{Parameters}

For assessment of the spines we used clinically relevant and methodologically validated parameters of lumbar spinal degeneration as recommended by the European Spine Society [12]. Grading methods for disc degeneration with an intraclass correlation coefficient or an interobserver $\kappa>0.60[9,17,20]$ were included. For facet joint degeneration, grading schemes [12] with an intraclass correlation coefficient or interobserver $\kappa>0.40$ were used in the present study $[19,27]$.

Magnetic resonance imaging (MRI, Siemens@ Symphony 1.5 T: Syngo MR A30, software NUMARIS/4, Berlin, Germany) of lumbar spines was performed to assess intervertebral disc degeneration according to Griffith [9] and Pfirrmann [20] and facet joint degeneration according to Weishaupt [27]. Disc degeneration [28], narrowing (Lane-1) [17], osteophytes (Lane-2) [17] and facet joint degeneration [19] of levels L2-L3 and L4-L5 were also assessed based on radiographs (Sedical@ $\odot$ Digital Vet. DX-6, Arlington Heights, IL, USA). Furthermore, MRI was used to assess the presence of Modic changes [18] and Schmorl's nodes [21] and to determine intervertebral disc and pedicle geometry and facet joint angles [5]. Disc geometry included: disc length, width, height, surface area, and volume. Disc surface area, disc volume and pedicle diameter were calculated assuming an elliptic shape $\quad($ surface $=1 / 4 \pi \times$ length $\times$ width $)$. For pedicle diameter, an average of left and right pedicles was taken for the top (L2 or L4) and bottom (L3 or L5) of each segment. Mean facet joint angle was calculated by averaging left and right angles per segmental level (L2-L3 or L4-L5), while facet angle differences or tropism was determined by calculating the difference between left and right facet joint angles. Segmental frontal surface area (FA), defined in $\mathrm{cm}^{2}$, bone mineral content (BMC, in $\mathrm{g}$ ) and bone mineral density (BMD, in $\mathrm{g} / \mathrm{cm}^{2}$ ) of lumbar spinal sections (L2-L3 and L4-L5) were measured with dual X-ray absorptiometry (DXA, Hologic $@$ QDR 4500 Delphi DXA scanner, Waltham, MA, USA) in anteroposterior direction. Specimens were dissected before they were scanned in a tank filled with saline $(0.9 \% \mathrm{NaCl})$. All assessments were performed using Osirix software (Osirix $\odot$, version 4.1.2., Pixmeo SARL, Geneva, Switzerland).

Specimen preparation and biomechanical testing

L2-L3 and L4-L5 motion segments were isolated from each spine. Subsequently, laminectomy was performed at level L2 of five randomly chosen spines, and at level L4 of the remaining five spines. Laminectomy, analogous to standard clinical practice, was performed by removing the spinous process and part of the lamina, leaving the facet joints intact. During preparation, examination, and biomechanical testing, specimens were kept hydrated using $0.9 \%$ saline-soaked gauzes. Thoracolumbar spines with 
bridging osteophytes, assessed on anteroposterior, lateral and oblique radiographs, were excluded from this study. After sectioning spines in L2-L3 and L4-L5 motion segments, the motion segments were potted in a casting-mould using low melting point $\left(48{ }^{\circ} \mathrm{C}\right.$ ) bismuth alloy (Cerrolow-147; $48.0 \%$ bismuth, $25.6 \%$ lead, $12 \%$ tin, $9.6 \%$ cadmium, and $4 \%$ indium). The upper and lower vertebral bodies were fixed securely into the alloy by adding screws into the vertebral body. Screw fixation was reinforced with orthopaedic bone cement (Simplex, Stryker@, Kalamazoo, MI, USA). The disc was placed parallel to the flat surface of the bismuth, based on visual inspection. Because muscle tissue was thoroughly and carefully removed, the intervertebral disc and corresponding endplates were clearly visible. All articulating parts were kept free. The casting-mould was placed in a hydraulic materials testing machine (Instron (C), model 8872, Norwood, Canada) [3]. The caudal vertebral body was fixed on a plateau that allowed movement in axial and transverse directions only. Transverse movements were allowed, so segments were able to find their physiological motion patterns and to correct for possible differences in embedding. Segments were loaded with a continuous axial compressive force of 1,600 N [2-4, 24, 26], a force that is compatible with lumbar spine compression forces that occur while moderately bending forward and low enough to avoid damage from compression alone [6]. The force was applied using a pneumatic cylinder that had been calibrated using a load cell (Hottinger Baldwin Messtechnik@), Force Transducer Type C2, Darmstadt, Germany). Since compression was applied in a purely axial direction, bending moments were minimized. Subsequently, while maintaining the axial load, torsion load was applied with a constant rate of $3.0^{\circ}$ per min through a cable, which was securely fixed to the part of the casting-mould that contained the caudal vertebral body [3]. The test was stopped after hearing a crack or after a large moment reduction was seen. Torsion moments and displacement were digitized and stored at 100 samples per second (Instron(C) Fast Track 2, Norwood, Canada).

For each of the 20 motion segments tested, TMF was determined. TMF was defined as the point at which maximum load was recorded in the load-displacement curves for each specimen. These data were presented previously [3]. Early torsion stiffness (ETS) and late torsion stiffness (LTS) were calculated from the load-displacement curve, between, respectively, 20-40 and 60-80\% of the TMF. ETS and LTS were estimated by means of a least squares fit of a straight line through the data with the slope of the regression line representing stiffness. All analyses were performed using computer programs written in Matlab (Mathworks (C), Natick, MA, USA).

Statistical methods

Statistical analysis was performed based on two separate groups. The first group contained untreated segments $(5 \times$
L2-L3 and 5× L4-L5), while the second group consisted of segments with laminectomy (5× L2-L3 and 5× L4-L5).

Independent variables were classified as: general variables, intervertebral disc geometry (MRI), pedicle geometry (MRI), facet joint orientation (MRI), bone characteristics (DXA), intervertebral disc degeneration classifications (MRI), intervertebral disc and facet joint degeneration (radiographs), facet joint degeneration (MRI) and other (MRI). These classes of variables are specified in Table 2.

First, relations between independent and dependent variables (ETS, LTS and TMF) were tested for each individual variable. For dichotomized independent variables (segment, sex, Modic changes [18] and Schmorl's nodes [21]), an independent-samples $t$ test was used, while Pearson's coefficient of correlation was determined for continuous and ordinal values. Note that it was thus assumed that ordinal variables (Pfirrmann [20], Griffith [9], Lane-1 [17], Lane-2 [17], Wilke [28], Pathria [19] and Weishaupt [27]) represent a linear degree of severity.

When independent variables were associated with a dependent variable, here defined as independent-samples $t$ test: $p<0.05$ or as a bivariate correlation with a significance level of $p<0.05$, they were used for the combined statistical models. Before final analysis was performed, all independent variables were checked for correlations with each other. In case a correlation $>0.7$ with a $p$ value $<0.05$ was found, the independent variable with the strongest correlation with the specific dependent variable was included in the model. Finally, backward linear regression was used to create a multivariate model per dependent variable per treatment group.

\section{Results}

Averaged biomechanical properties for segments with and without laminectomy are presented in Table 1. Data of individual segments were presented previously [3].

Table 2 gives an overview of associations between independent and dependent variables of segments with and without laminectomy. Only two of the general variables were related to biomechanical outcomes, i.e. segment for ETS in the untreated group and sex for LTS of treated segments. In the untreated segments, ETS was lower for L4-L5 than for L2-L3 (8.9 versus $\left.12.6 \mathrm{~N} \mathrm{~m} /{ }^{\circ}\right)$. LTS in treated segments proved to be sex dependent (male 4.1 versus female $3.7 \mathrm{~N} \mathrm{~m} /{ }^{\circ}$ ).

In segments with laminectomy, only ETS was related to intervertebral disc geometry variables (i.e. width and surface). In untreated segments, only TMF was related to intervertebral disc geometry (i.e. width and surface). Pedicle geometry of the top levels (i.e. L2 and L4) after laminectomy was related to ETS and TMF. Pedicle 
Table 1 Overview of biomechanical outcomes

\begin{tabular}{|c|c|c|c|c|c|c|}
\hline & \multicolumn{2}{|c|}{ Early torsion stiffness $(\mathrm{ETS})\left(\mathrm{Nm} /{ }^{\circ}\right)$} & \multicolumn{2}{|c|}{ Late torsion stiffness $(\mathrm{LTS})\left(\mathrm{Nm} /{ }^{\circ}\right)$} & \multicolumn{2}{|c|}{ Torsion moment to failure (TMF) (Nm) } \\
\hline & Absolute $(+\mathrm{SD})$ & Percentage difference & Absolute (+SD) & Percentage difference & Absolute $(+\mathrm{SD})$ & Percentage difference \\
\hline Untreated & $10.8(5.4)$ & $34.1 \%$ & $5.7(5.0)$ & $30.1 \%$ & $51.4(14.7)$ & $17.6 \%$ \\
\hline Laminectomy & $7.1(4.1)$ & & $4.0(2.9)$ & & $42.4(17.5)$ & \\
\hline
\end{tabular}

Early and late stiffness were estimated over $20-40 \%$ and $60-80 \%$ of the torsion moment to failure, respectively, and moment-deformation curves were linear within these ranges, with $r^{2}$ values between load and displacement all above 0.96 except for four values

Bold values indicate a significance level at $p<0.05$

geometry of the lower levels (i.e. L3 and L5) was related to TMF in untreated segments. Facet angle difference in treated segments was correlated to both stiffness parameters (i.e. ETS and LTS).

Bone characteristics measured with DXA were strongly related with all biomechanical parameters after laminectomy. Specifically, segmental frontal area and BMC were significantly correlated to spinal biomechanics. In untreated segments, BMC was only correlated to TMF.

In contrast to DXA parameters, intervertebral disc degeneration was only predictive for biomechanics (ETS and LTS) of spinal segments without laminectomy. Furthermore, this mainly applied to MRI and radiographic classification schemes (Pfirrmann [20], Griffith [9], and Lane-1 [17]). No correlation between disc degeneration and spinal strength (TMF) was established. Finally, Modic changes [18] and Schmorl's nodes [21] were only related to ETS properties of spinal segments without laminectomy.

Results of the backward linear regression, using determinants of spine biomechanics, which were identified (based on a $p$ value $<0.05$ ) in Table 2 , are presented in Table 3. All biomechanical parameters (ETS, LTS and TMF) could accurately be predicted by the final statistical model for segments treated with laminectomy $\left(r^{2}\right.$ values $0.95,0.87$ and 0.93 , respectively). Intervertebral disc width, facet angle difference and BMC remained in the model for ETS, while for LTS, the final model consisted of the same parameters without the addition of intervertebral disc width. For TMF segmental frontal area and BMC defined the final model. For untreated segments, moderately predictive models for ETS, LTS and TMF were defined with $r^{2}$ values of $0.69,0.61$ and 0.45 , respectively. The model for ETS consisted of Pfirrmann [20] and Modic changes [18] as variables, while LTS was predicted by Griffith [9]. Finally, the model for TMF consisted of pedicle geometry of the lower level.

\section{Discussion}

The aim of this study was to identify parameters that are correlated to spinal torsion properties, to develop a prediction model for spinal torsion instability following lumbar laminectomy.
We showed that multiple variables are related to spinal torsion properties in intact lumbar segments and in lumbar segments treated with laminectomy. Statistical models with these parameters as independent variables predicted torsion biomechanics, with moderate accuracy in untreated segments $\left(r^{2}\right.$ values $\left.0.45-0.69\right)$ and with very good accuracy $\left(r^{2}\right.$ values $\left.0.87-0.95\right)$ in treated segments.

For both treated and untreated segments, univariate associations between biomechanical behaviour and intervertebral disc geometry (i.e. disc width and disc surface) were found. It was striking that, in untreated segments, these geometrical parameters were significantly correlated to TMF, while in treated segments, a correlation with ETS was found. This possibly reflects the fact that laminectomy causes a shift in load-bearing from the posterior elements to the anterior elements [11], leading to a significant correlation between stiffness in the early phase and intervertebral disc geometry.

In untreated segments (Table 2), Pfirrmann [20] and Griffith [9] were correlated with stiffness properties (ETS and LTS), while in segments with laminectomy, no correlation was established with disc degeneration scales. While it could be argued that a difference in degeneration scores between treated and untreated segments could underlie the presence of degeneration parameters for untreated segments but not for treated segments, the data do not support this suggestion. Average degeneration scores were slightly higher for the non-treated segments, however, the standard deviation of scores was larger in treated segments.

In this study, we differentiated results between ETS and LTS in order to separately quantify stiffness in the early and late phase of the load-displacement curve. The transition zone between ETS and LTS in load-displacement curves possibly indicates yielding. This yield phase reflects a decrease in stiffness, which possibly indicates the first damage to the structure [23]. Since the yield phase in the load-displacement curves did not show a smooth continuous curve between ETS and LTS, we could not define a specific yield point as was defined previously for shear loading [4]. We expect that when a spinal segment reaches its LTS zone, sub-clinical damage will have occurred. Such damage may, at a later stage, lead to symptomatic 


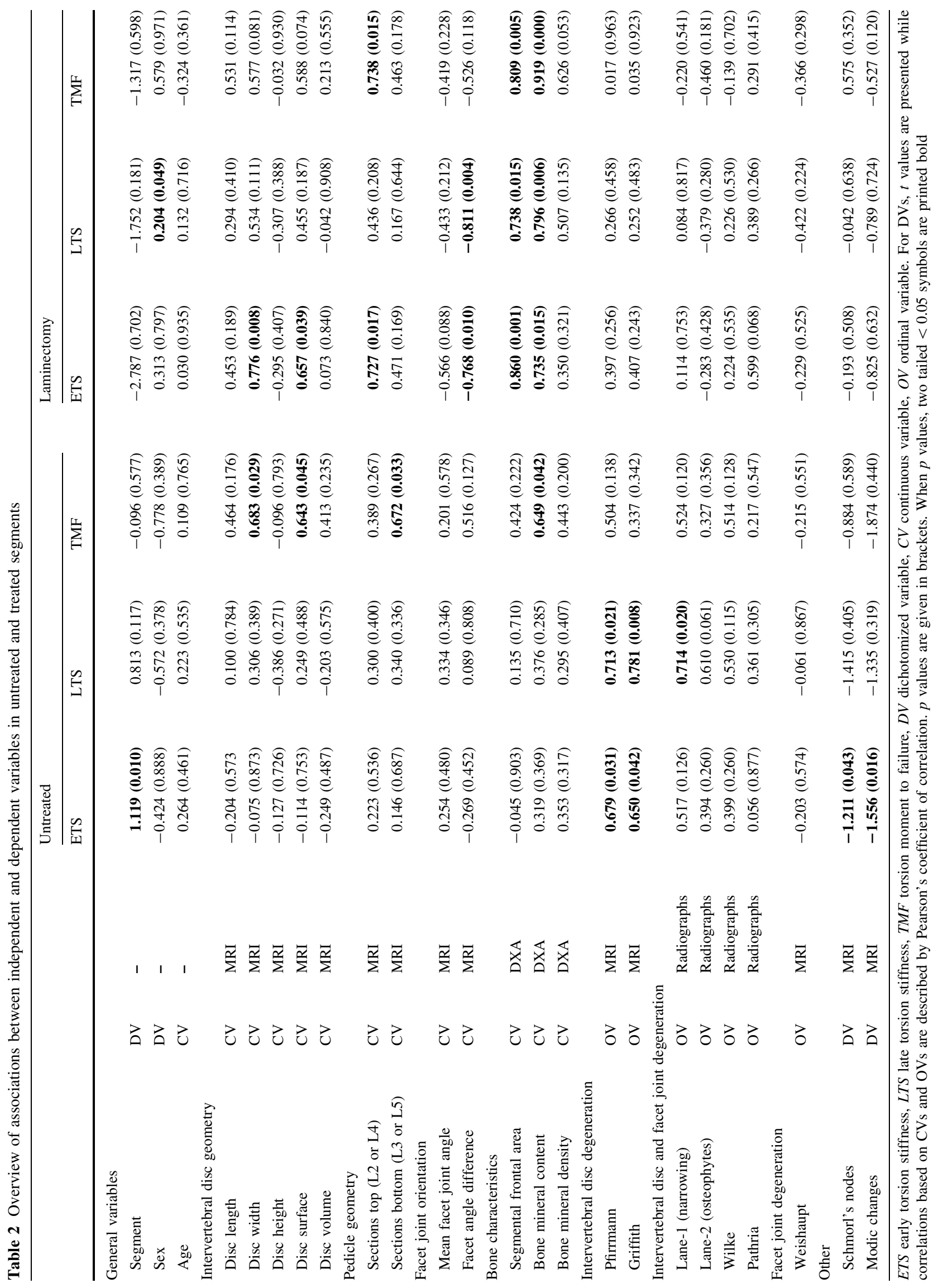


Table 3 Overview of backward linear regression models per dependent variable in untreated segments, based on significant correlation coefficients found in Table 2

\begin{tabular}{|c|c|c|c|c|c|}
\hline \multicolumn{6}{|l|}{ Untreated } \\
\hline Early torsion stiffness & Variables: & Constant & Pfirrmann & Modic changes & \\
\hline $\mathrm{Nm} /{ }^{\circ}$ & Factor: & $-13.550(6.902)$ & $5.750(1.773)$ & $4.900(2.127)$ & \\
\hline$r^{2}$ value: 0.693 & Significance: & 0.090 & 0.014 & 0.055 & \\
\hline Late torsion stiffness & Variables: & Constant & Griffith & & \\
\hline $\mathrm{Nm} /{ }^{\circ}$ & Factor: & $-9.383(4.394)$ & $2.746(0.775)$ & & \\
\hline$r^{2}$ value: 0.610 & Significance: & 0.065 & 0.008 & & \\
\hline Torsion moment to failure & Variables: & Constant & Pedicle sections bottom & & \\
\hline $\mathrm{Nm}$ & Factor: & $26.430(10.380)$ & $25.938(10.100)$ & & \\
\hline$r^{2}$ value: 0.452 & Significance: & 0.034 & 0.033 & & \\
\hline \multicolumn{6}{|l|}{ Laminectomy } \\
\hline Early torsion stiffness & Variables: & Constant & Intervertebral disc width & Facet angle difference & $\mathrm{BMC}$ \\
\hline $\mathrm{Nm} /{ }^{\circ}$ & Factor: & $-18.618(4.921)$ & $3.489(0.721)$ & $-0.461(0.135)$ & $0.174(0.046)$ \\
\hline$r^{2}$ value: 0.952 & Significance: & 0.009 & 0.003 & 0.014 & 0.009 \\
\hline Late torsion stiffness & Variables: & Constant & Facet angle difference & $\mathrm{BMC}$ & \\
\hline $\mathrm{Nm} /{ }^{\circ}$ & Factor: & $1.219(1.817)$ & $-0.493(0.137)$ & $0.166(0.049)$ & \\
\hline$r^{2}$ value: 0.871 & Significance: & 0.524 & 0.009 & 0.011 & \\
\hline Torsion moment to failure & Variables: & Constant & Segmental frontal area & $\mathrm{BMC}$ & \\
\hline $\mathrm{Nm}$ & Factor: & $-30.540(9.156)$ & $0.898(0.298)$ & $1.300(0.242)$ & \\
\hline$r^{2}$ value: 0.932 & Significance: & 0.012 & 0.019 & 0.001 & \\
\hline
\end{tabular}

Each row in the table represents a regression equation

spondylolisthesis and/or rotoscoliosis [16]. Unlike LTS, TMF marks, as the description suggests, complete and irreversible failure of spinal motion segments. TMF describes an acute clinically relevant situation. Therefore, TMF and shear force to failure represent different clinical value.

For shear biomechanical prediction, DXA parameters, including BMD, BMC and segmental frontal area, were found to be of utmost importance [4]. However, in the present study, only BMC and segmental frontal area were found to be important in the prediction of torsion stiffness parameters after laminectomy. In our protocol, both BMD and BMC were studied. BMD is often used as a clinical parameter. However, BMC can also be used to express the bone mineral content since it integrates information on bone density and vertebral dimensions. BMC is defined as BMD $\left(\mathrm{g} / \mathrm{cm}^{2}\right)$ multiplied by the total segmental surface area (FA) of the spinal segment $\left(\mathrm{cm}^{2}\right)$ and is expressed in grams. Based on our results we conclude that BMC is a more useful parameter in predicting spinal biomechanical properties. Low BMC may thus be seen as an indication for using instrumentation after laminectomy. Note, however, also pull out strength of spinal implants proved to be dependent on bone mineral quality as measured by DXA [25] and this dependency needs to be taken into account when deciding on additional instrumentation.
In vivo, muscle forces generate substantial compression forces on the spine [22]. We simulated this by applying a static 1,600 N compressive force. Mainly due to muscle forces, the spine is already subjected to forces of this magnitude when the trunk is inclined about $45^{\circ}$ forward. When lifting a $10 \mathrm{~kg}$ object from ground level, compression forces can increase up to about 5,000 N [13]. Failure compressive loads in human cadaveric spines are on average 3,000 $\mathrm{N}$ [6]. The compression load of 1,600 $\mathrm{N}$ was also selected to allow for comparison with previous work $[2-4,24,26]$ and was sufficiently large to simulate physiological loading [13-15], but low enough to avoid damage due to compression forces alone [6].

Finally, while $r^{2}$ values, as we presented for untreated segments, may be too low ( $r^{2}$ values $\left.0.45-0.69\right)$ to provide clinically valuable data for the untreated segment, biomechanical parameters in treated segments were predicted with high accuracy $\left(r^{2}\right.$ values $\left.0.87-0.95\right)$. At present, surgeons decide based upon personal experience whether to apply instrumented stabilization after laminectomy. Our prediction models for laminectomy segments suggest that clinical decision-making may benefit from taking variables into account that are readily available from DXA measurements, i.e. segment frontal area and BMC. Considering further research, we recommend to assess the parameters, 
found to be predictive here, in a prospective or retrospective in vivo design, to define true clinical value. Furthermore, we were not able to obtain younger specimen with high DXA scores and completely healthy discs for our study. Therefor we must acknowledge that our data applies to the elderly population and is may not hold for younger subjects.

In conclusion, predictive models, mainly based on DXA variables, were found to accurately predict rotational biomechanical behaviour of the human lumbar spinal segments treated with laminectomy. In contrast, biomechanical behaviour of untreated segments was only moderately predicted. Proposed diagnostic parameters might aid surgical decision-making when deciding upon the use of instrumentation techniques.

\section{Conflict of interest None.}

\section{References}

1. Abel MS (1989) Transverse posterior element fractures associated with torsion. Skelet Radiol 17:556-560

2. Bisschop A, Mullender MG, Kingma I, Jiya TU, van der Veen AJ, Roos JC, van Dieen JH, van Royen BJ (2012) The impact of bone mineral density and disc degeneration on shear strength and stiffness of the lumbar spine following laminectomy. Eur Spine $\mathbf{J}$ 21:530-536

3. Bisschop A, van Dieen JH, Mullender M, Paul CPL, Jiya TU, Kingma I, van der Veen A, De Kleuver M, van Royen BJ (2013) Torsion biomechanics of the spine following lumbar laminectomy: a human cadaver study. Eur Spine J 22:1785-1793

4. Bisschop A, van Royen BJ, Mullender MG, Paul CP, Kingma I, Jiya TU, van der Veen AJ, van Dieen JH (2012) Which factors prognosticate spinal instability following lumbar laminectomy? Eur Spine J 21:2640-2648

5. Boden SD, Riew KD, Yamaguchi K, Branch TP, Schellinger D, Wiesel SW (1996) Orientation of the lumbar facet joints: association with degenerative disc disease. J Bone Joint Surg Am 78:403-411

6. Brinckmann P, Biggemann M, Hilweg D (1989) Prediction of the compressive strength of human lumbar vertebrae. Spine (Phila Pa 1976) $14: 606-610$

7. Farfan HF (1984) The torsional injury of the lumbar spine. Spine (Phila Pa 1976) 9:53

8. Gordon SJ, Yang KH, Mayer PJ, Mace AH Jr, Kish VL, Radin EL (1991) Mechanism of disc rupture. A preliminary report. Spine (Phila Pa 1976) 16:450-456

9. Griffith JF, Wang YX, Antonio GE, Choi KC, Yu A, Ahuja AT, Leung PC (2007) Modified Pfirrmann grading system for lumbar intervertebral disc degeneration. Spine (Phila Pa 1976) 32:E708E712

10. Gunzburg R, Hutton W, Fraser R (1991) Axial rotation of the lumbar spine and the effect of flexion. An in vitro and in vivo biomechanical study. Spine (Phila Pa 1976) 16:22-28
11. Haher TR, O'Brien M, Felmly WT, Welin D, Perrier G, Choueka J, Devlin V, Vassiliou A, Chow G (1992) Instantaneous axis of rotation as a function of the three columns of the spine. Spine (Phila Pa 1976) 17:S149-S154

12. Kettler A, Wilke HJ (2006) Review of existing grading systems for cervical or lumbar disc and facet joint degeneration. Eur Spine J 15:705-718

13. Kingma I, Bosch T, Bruins L, van Dieen JH (2004) Foot positioning instruction, initial vertical load position and lifting technique: effects on low back loading. Ergonomics 47:1365-1385

14. Kingma I, Faber GS, Bakker AJ, van Dieen JH (2006) Can low back loading during lifting be reduced by placing one leg beside the object to be lifted? Phys Ther 86:1091-1105

15. Kingma I, Staudenmann D, van Dieen JH (2007) Trunk muscle activation and associated lumbar spine joint shear forces under different levels of external forward force applied to the trunk. J Electromyogr Kinesiol 17:14-24

16. Kirkaldy-Willis WH, Farfan HF (1981) Instability of the lumbar spine. Clin Orthop Relat Res 165:110-123

17. Lane NE, Nevitt MC, Genant HK, Hochberg MC (1993) Reliability of new indices of radiographic osteoarthritis of the hand and hip and lumbar disc degeneration. J Rheumatol 20:1911-1918

18. Modic MT, Steinberg PM, Ross JS, Masaryk TJ, Carter JR (1988) Degenerative disk disease: assessment of changes in vertebral body marrow with MR imaging. Radiology 166:193-199

19. Pathria M, Sartoris DJ, Resnick D (1987) Osteoarthritis of the facet joints: accuracy of oblique radiographic assessment. Radiology 164:227-230

20. Pfirrmann CW, Metzdorf A, Zanetti M, Hodler J, Boos N (2001) Magnetic resonance classification of lumbar intervertebral disc degeneration. Spine (Phila Pa 1976) 26:1873-1878

21. Pfirrmann CW, Resnick D (2001) Schmorl's nodes of the thoracic and lumbar spine: radiographic-pathologic study of prevalence, characterization, and correlation with degenerative changes of 1,650 spinal levels in 100 cadavers. Radiology 219:368-374

22. Quint U, Wilke HJ, Loer F, Claes L (1998) Laminectomy and functional impairment of the lumbar spine: the importance of muscle forces in flexible and rigid instrumented stabilization-a biomechanical study in vitro. Eur Spine J 7:229-238

23. Renau A, Farrerons J, Yoldi B, Gil J, Proubasta I, Lalauger J (2004) Precision of bone mineral density scans at the proximal tibia in osteoarthritic subjects. J Clin Densitom 7:382-389

24. van Dieen JH, van der Veen A, van Royen BJ, Kingma I (2006) Fatigue failure in shear loading of porcine lumbar spine segments. 8 31:E494-E498

25. van Laar W, Meester RJ, Smit TH, van Royen BJ (2007) A biomechanical analysis of the self-retaining pedicle hook device in posterior spinal fixation. Eur Spine J 16:1209-1214

26. van Solinge GB, van der Veen AJ, van Dieen JH, Kingma I, van Royen BJ (2010) Anterior shear strength of the porcine lumbar spine after laminectomy and partial facetectomy. Eur Spine J 19:2130-2136

27. Weishaupt D, Zanetti M, Boos N, Hodler J (1999) MR imaging and CT in osteoarthritis of the lumbar facet joints. Skelet Radiol 28:215-219

28. Wilke HJ, Rohlmann F, Neidlinger-Wilke C, Werner K, Claes L, Kettler A (2006) Validity and interobserver agreement of a new radiographic grading system for intervertebral disc degeneration: part I. Lumbar spine. Eur Spine J 15:720-730 